\title{
Physically consistent metrics with independent parameters instead of standard metrics with unphysical consequences. 1. Charged sources
}

\author{
Zahid Zakir ${ }^{1}$
}

\begin{abstract}
The metric around a ball with a surface charge, obtained from the Einstein-Maxwell equations, has three independent parameters - the charge, surface radius and the ball's gravitational radius, equal to the gravitational radius of the matter. The electrostatic energy there is only outside the ball and contributes to the metric with the same sign as matter. Therefore, an increase in charge enhances the effects of gravity, increasing redshifts, the radii of orbits and shadow. At the collapse, in the rest frame of the center, the surface freezes above the ball's gravitational radius, and the inner layers freeze above the gravitational radius of the matter inside them, i.e. the collapsed ball becomes not a black hole, but a frozar, an object with a gravitationally frozen structure. The frozar's metric follows from the ball's metric as its surface tends to the gravitational radius and thus contains two parameters instead of three. The frozar's charge freezes over the gravitational radius and its total mass, as for the ball, is finite. Observable consequences of the frozar metric are the same as for the ball. On the contrary, the Reissner-Nordström metric, used in the black hole theory, contains the total mass at infinity, which depends on the charge and diverges, since it includes the energy of the electric field diverging for a point charge. To ignore this divergence, the total mass was renormalized and replaced with an "observable" mass. Then, already erroneously, the dependence of the total mass on the charge was missed. The result of this double disregard was a set of unphysical consequences, inverse to the ball, which complicated the physical picture. It was argued that there are two horizons and the increase in charge weakens the gravity by decreasing the gravitational radius and observable effects of gravity, such as redshifts, radii of orbits and shadow, i.e. it was seriously stated that the positive energy of the electric field antigravitates, which is physically absurd. Other nonphysical aspects of the black hole theory are also discussed.
\end{abstract}

Keywords: charged ball, gravitational radius, Reissner-Nordström metric, frozar, black hole, effects of gravity, redshift, shadow, light deflection, gravitational collapse

\section{Content}

1. Introduction 1

2. A solution of the Einstein equations for a charged dust ball ........................................................... 3

3. The external metrics for a charged frozar and its properties................................................................. 5

4. The time dilation factor and redshifts near compact charged sources..................................................... 7

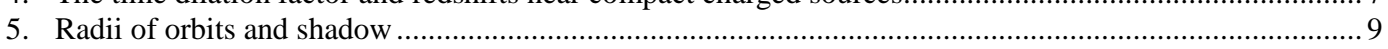

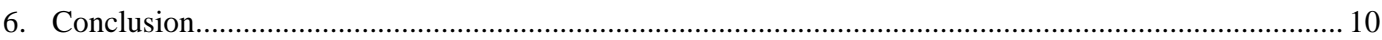

Appendix. The unphysical character of the black hole theory and its consequences ...................................... 11

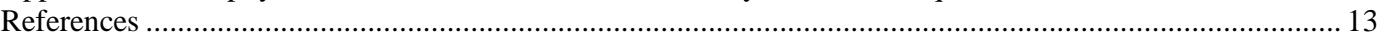

\section{Introduction}

A general form of an exact solution of the Einstein-Maxwell equations outside a spherical source of charge $e$ was found in 1916-1918 by Reissner [1], Weyl [2] and Nordström [3] (see also [4]) and included the total mass of the source at infinity. The obtained metric, called the Reissner-Nordström (RN) metric, had the form:

\footnotetext{
${ }^{1}$ Center for Theoretical Physics and Astrophysics, Tashkent Uzbekistan, zzakir@qgph.org, ORCID
} 


$$
g_{00}(r)=-g_{11}^{-1}(r)=1-\frac{\bar{r}_{g}}{r}+\frac{Q^{2}}{r^{2}},
$$

where $Q^{2}=G e^{2} / c^{4}, G$ - gravitational constant, and $\bar{r}_{g}$ is an integration constant.

Here $\bar{r}_{g}$ is the effective gravitational radius $\bar{r}_{g}=2 G \bar{M} / c^{2}$ associated with the total mass at infinity $\bar{M}$, determined from correspondence in the asymptotics with the Schwarzschild metric. In the black hole theory, the RN metric (1) was considered as a solution for a point source with two independent parameters $\bar{M}$ and $Q$ [3-6] (Fig. 1). This led to further unphysical consequences, such as the complicated structure of space-time with two horizons, and the predictions that an increase in $Q$ weakens gravity by decreasing the gravitational radius and observable effects of gravity, in particular redshifts, orbital and shadow radii $[5,10]$. But $\bar{M}$ includes the total energy of the electric field and increases with the increase in $Q$. The omission of this fact was a mistake that led to these unphysical consequences and predictions.

The situation when the positive energy of electric field antigravitates was fundamentally unacceptable. To exclude this, Einstein was even ready to change the sign of the electric field energy in his equations and in 1935 he and Rosen seriously proposed this in [6]. In this article, it was also stated without proof that $\bar{M}$ and $Q$ in (1) are independent constants.

Thus, the physical consequences of the RN metric (1) depended on the explicit form of the constant $\bar{r}_{g}$, taking into account its physical meaning. The correct solution to this problem for a ball with a charge in entire volume, but with mutually dependent parameters, was given even in 1960 by W. Bonnor [7] and P. Florides [8] (Fig. 2). They found the dependence $\bar{M}=M_{b}+e^{2} /\left(2 r_{b} c^{4}\right)$, where $r_{b}$ is the radius of the ball's surface and $M_{b}$ is its total mass without energy of the outer electric field, and showed that the growth of the charge enhances gravity around the ball (see also [9]). However, the dependence of $M_{b}$ on the internal charge density complicated the solution, since the gravitational radius of the ball $r_{g b}=2 G M_{b} / c^{2}$, figured in the modified metric, remains dependent on $Q$. As the result, in the metric, one dependent on $Q$ the parameter, $\bar{r}_{g}$, was replaced by another, $r_{g b}$, also dependent one.

In the present article, the external metric for the ball will be obtained for a more realistic case when the charge there is on the surface only (Fig. 3). In this case $M_{b}$ is independent on $Q$ and the metric with three independent parameters $r_{b}, r_{g}$ and $Q$ takes the form:

$$
g_{00}(r)=-g_{11}^{-1}(r)=1-\frac{r_{g}}{r}-\frac{Q^{2}}{r}\left(\frac{1}{r_{b}}-\frac{1}{r}\right)=1-\frac{r_{g}}{r}-\frac{Q^{2}}{r_{b} r}\left(1-\frac{r_{b}}{r}\right), \quad r \geq r_{b},
$$

where $r_{g}=2 G M / c^{2}$ - gravitational radius of matter in the ball with mass $M$ and $r_{b}>r_{g}$.

In the articles [11], the gravitational field and structure of a neutral dust ball were studied according to the Oppenheimer-Snyder exact solution [12] and it was shown that in the rest frame of the center the general relativistic collapse leads to the formation of a frozar, a gravitationally frozen object, the surface of which is frozen above the gravitational radius and $r_{b} \rightarrow r_{g}$ at $c t \gg r_{g}$. The inner layers of the ball also freeze near the gravitational radius of matter inside them. The metric for the charged frozar follows from the metric for the charged ball (2) at $r_{b} \rightarrow r_{g}$ (Fig. 4) and has the form: 


$$
g_{00}(r)=-g_{11}^{-1}(r) \simeq\left(1-\frac{r_{g}}{r}\right)\left(1-\frac{Q^{2}}{r_{g} r}\right)=1-\frac{r_{g}+Q^{2} / r_{g}}{r}+\frac{Q^{2}}{r^{2}}, \quad r>r_{g}
$$

Unlike (2), this metric contains only two independent parameters - $r_{g}$ and $Q$.

In both metrics (2) and (3), an increase in $Q$ strengthens the gravity, which leads to a physically correct dependence of the observable effects of gravity on $Q$. In particular, redshifts, orbital and shadow radii increase with the increase in $Q$ (Figs. 5,7).

Eqs. (2)-(3) show that ignoring the dependence of $\bar{M}$ on $Q$ was not a casual, since in the black hole theory, the divergence of electrostatic energy of a point charge leads to a divergent total mass. A proposal to include this dependence as $\bar{M}=M+e^{2} /\left(4 M G c^{2}\right)$ [9] did not lead to a revision of the RN metric (1), since for the black hole as a point source there is no physical reason for cutting off the electric field at the gravitational radius.

In the frozar theory, the charge is almost at $r_{g}$ and even if we proceed from the RN metric (1), then the contribution of the total electrostatic energy to $\bar{M}$ will be equal to $Q^{2} / r_{b} \rightarrow Q^{2} / r_{g}$, and the frozar metric (3) follows from (1) at replacing of $\bar{r}_{g}$ as:

$$
\bar{r}_{g}=r_{g}+\frac{Q^{2}}{r_{b}} \simeq r_{g}+\frac{Q^{2}}{r_{g}}
$$

This agrees with the proposals in [7-9], which is explained by the fact that the purely physical arguments presented in them are natural precisely in the frozar theory.

In the article it will be shown that taking into account the dependence of $\bar{r}_{g}$ on $Q$ in the form (4) leads to correct physical consequences. In this case, to obtain the results of calculating the observable physical effects in the frozar theory, it is enough to make the substitution (4) in all the corresponding formulas with the RN metric (1). Despite such a simple formal difference, the character of the dependence of the observable effects on $Q$ in the frozar theory becomes opposite to the results that were previously predicted in the black hole theory (Figs. 5-8).

Thus, the metrics (2)-(3) for the realistic case of a ball with a surface charge, both in normal and collapsed states, are physically consistent metrics with independent parameters for a charged source, and this completes more than a century of very dramatic history of this problem.

In Section 2 of the article, the solution for the external metrics for the charged dust ball is presented, and in Section 3, the metric for the charged frozar is studied. In Sections 4 and 5 some of the observable effects in the field of a charged frozar are described. In the Appendix, an unphysical character of the black hole theory and its consequences are considered.

\section{A solution of the Einstein equations for a charged dust ball}

The spacetime interval inside and outside the charged dust ball on the hypersurfaces of simultaneity $t=$ const can be reduced to the diagonal form:

$$
d s^{2}=e^{v} c^{2} d t^{2}-e^{\lambda} d r^{2}-r^{2} d \Omega^{2}
$$

where $d \Omega^{2}=d \theta^{2}+\sin ^{2} \theta d \varphi^{2}$. For a neutral ball, the metric in (5) at $r \geq r_{b}$ is given by the Schwarzschild solution $e^{v(r)}=e^{-\lambda(r)}=1-r_{g} / r$, where $r_{g}=2 G M / c^{2}$ is the gravitational radius of the ball.

A charged ball as a source of gravity, unlike a neutral one, is non-compact, since it is not localized inside its surface $r_{b}$, containing matter with the energy density $\rho_{M}$. There is a 
fundamentally new aspect - the ball's total mass of the source also includes the mass-equivalent of the electrostatic energy in a sphere of radius $r>r_{b}$ and therefore depends on $r$ and $Q$. This electrostatic energy also depends on the charge density in the source (Fig. 1) [7,8]. In the case of a point charge (charged black hole) (Fig. 2), the electrostatic energy diverges and therefore the total mass also diverges.

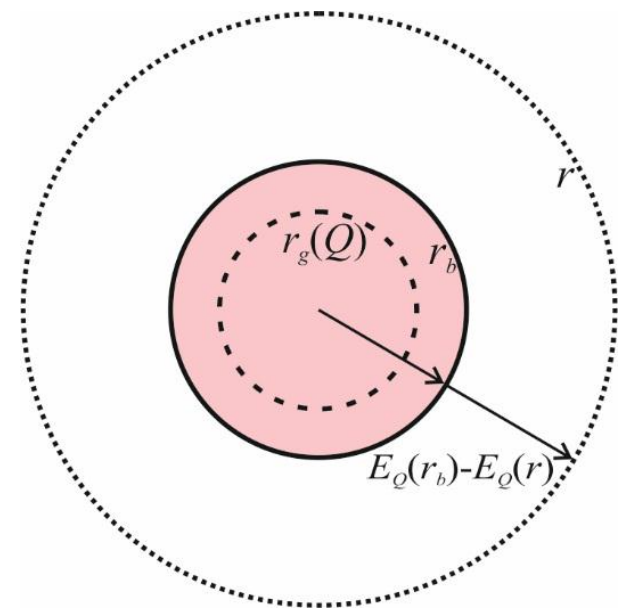

Fig. 1. Gravitational and electric fields of a ball with a volume charge $[7,8]$.

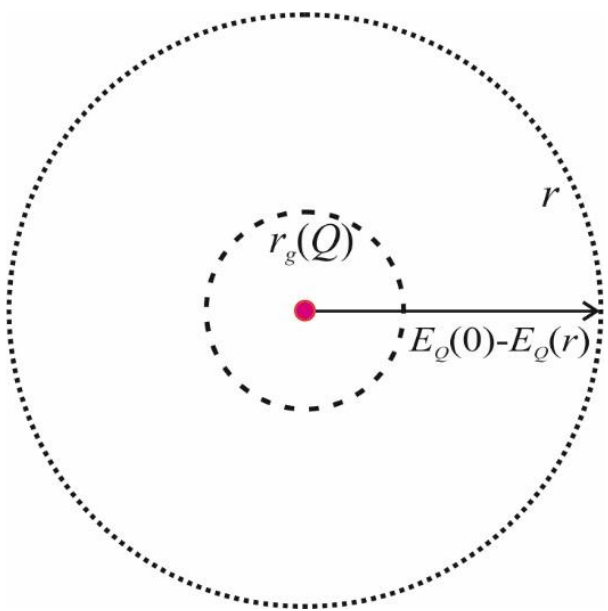

Fig. 2. Gravitational and electric fields of a point charge (black hole) [1-6].

In the case of a real charged ball with $r_{b} \gg r_{g}$, the electrostatic equilibrium occurs only when the charge becomes collected on the surface and this ensues quickly. This fact simplifies the situation with the calculation of electrostatic energy, since its energy density $\rho_{e}$ becomes nonzero only on the surface and in the outer region $\left(r_{b} \leq r<\infty\right)$. Now $\rho_{e}$ contributes only to the external metric, $\rho_{M}$ contributes only to the internal metric $\left(0 \leq r<r_{b}\right)$, and on the surface $\left(r=r_{b}\right.$ ) both metrics are matched (Fig. 3).

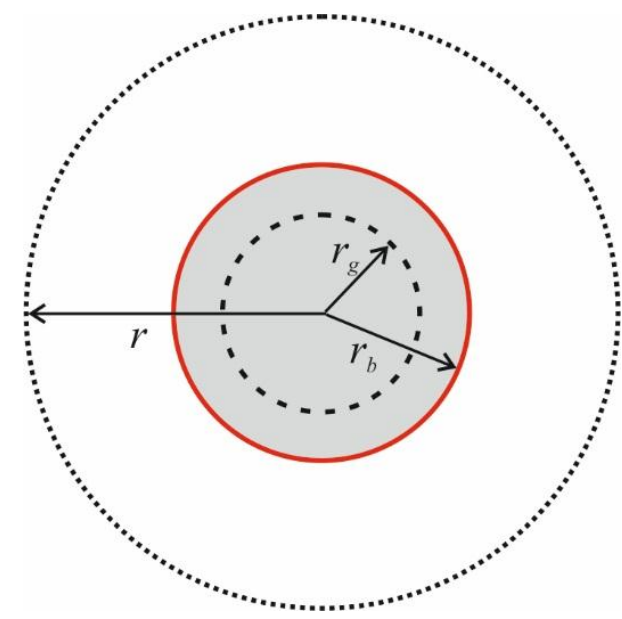

Fig. 3. Gravitational and electric fields of a ball with a surface charge.

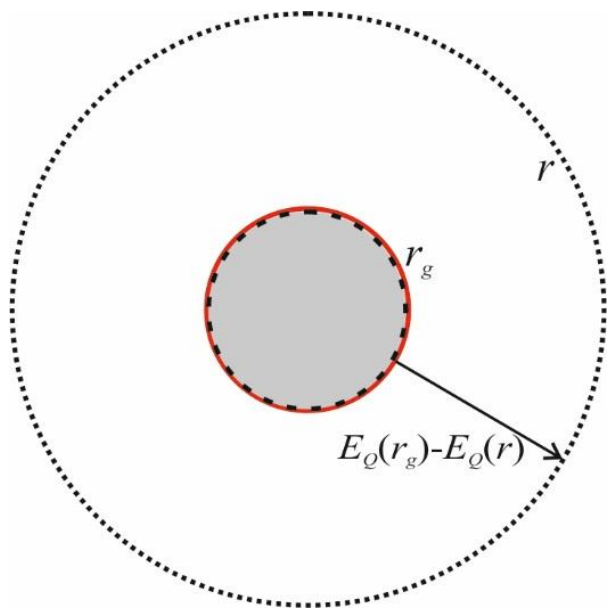

Fig. 4. Gravitational and electric fields of a frozar with a surface charge $\left(r_{b} \rightarrow r_{g}\right)[11,14]$. 
The Einstein equations with such separate contributions from two regions take the form:

$$
\begin{aligned}
& -e^{-\lambda}\left(\frac{1}{r^{2}}+\frac{v^{\prime}}{r}\right)+\frac{1}{r^{2}}=\kappa T_{1}^{1}=\kappa \theta\left(r-r_{b}\right) \rho_{e}, \quad \kappa=8 \pi G / c^{4}, \\
& -e^{-\lambda}\left(\frac{1}{r^{2}}-\frac{\lambda^{\prime}}{r}\right)+\frac{1}{r^{2}}=\kappa T_{0}^{0}=\kappa\left[\theta\left(r_{b}-r\right) \rho_{M}+\theta\left(r-r_{b}\right) \rho_{e}\right],
\end{aligned}
$$

In the outer region $r>r_{b}$, where $\rho_{M}=0$, subtracting (6) from (7) gives $v^{\prime}+\lambda^{\prime}=0$, which means that $v=-\lambda+f, f^{\prime}=0$ and, after redefining $d t^{\prime}=e^{f} d t$, gives $e^{v}=e^{-\lambda}$.

Further, we rewrite equation (7) in the form:

$$
\left(r e^{-\lambda}\right)^{\prime}=1-\kappa\left[\theta\left(r_{b}-r\right) \rho_{M}+\theta\left(r-r_{b}\right) \rho_{e}\right] r^{2}
$$

and reduce the integral in the interval $(0, r)$ to two independent integrals in the intervals $\left(0, r_{b}\right)$ and $\left(r_{b}, r\right)$. Then, substituting for $\rho_{e}$ and the mass of matter $M$ their expressions [1-5]:

$$
\rho_{e}=\frac{e^{2}}{8 \pi r^{4}}, \quad M=\frac{4 \pi}{c^{2}} \int_{0}^{r_{b}} \rho_{M} r^{2} d r
$$

we come to the solution for $e^{-\lambda}$ :

$$
e^{-\lambda}=1-\frac{\kappa}{r}\left(\int_{0}^{r_{b}} \rho_{M} r^{2} d r+\frac{e^{2}}{8 \pi} \int_{r_{b}}^{r} \frac{d r}{r^{2}}\right)=1-\frac{r_{g}}{r}-\frac{Q^{2}}{r}\left(\frac{1}{r_{b}}-\frac{1}{r}\right) .
$$

The required external metric for the charged ball, thus, takes the form:

$$
e^{v}=e^{-\lambda}=1-\frac{r_{g}}{r}-\frac{Q^{2}}{r_{b} r}\left(1-\frac{r_{b}}{r}\right), \quad r \geq r_{b}>r_{g}
$$

On the surface and outside the ball $r \geq r_{b}>r_{g}$, this metric has no singularities and includes three independent parameters $r_{b}, r_{g}$ and $Q$. In this case, the expression in parentheses in (11) is positive definite and the contribution to the metric of the electric field's energy, proportional to $Q^{2}$, has the same sign as the contribution of the matter's energy. Therefore, an increase in the ball's charge $Q$ enhances its gravitational field at a point $r>r_{b}$ due to an increase in the electric field energy inside the sphere of radius $r$.

Therefore, observable effects of gravity, in particular, the radii of the orbits and shadow, redshifts and angles of deflection of particles in the field of the charged ball increase with increasing $Q$. The corresponding formulas and graphs are presented in Sections 4 and 5 in the simpler case of a frozar, when $r_{b} \simeq r_{g}$.

\section{The external metrics for a charged frozar and its properties}

In the preceding section, it was obtained the external metric (11) for a dust ball with a surface radius $r_{b}>r_{g}$ and a surface charge $e$. This metric contains three independent parameters $r_{b}, r_{g}$ and $Q$, which distinguishes it from the metric of a ball with a volume charge [7,8], where one of the parameters depends on the other two: $r_{g}\left(r_{b}, Q\right)$. 
At the gravitational collapse of the ball, its surface, in the rest frame of its center, asymptotically approaches $r_{g}$, i.e. $r_{b} \rightarrow r_{g}$ at $c t \gg r_{g}$. Such a collapsed ball, therefore, becomes a frozar, an object with a completely frozen structure [11] (Fig. 4). Any inner layer also freezes near the gravitational radius of matter inside it. Since $r_{b} \simeq r_{g}$, the frozar metric contains only two independent parameters $r_{g}$ and $Q$.

At collapse of the ball with the surface charge, the particles of neutral matter under the surface fall radially along geodesic trajectories, and therefore this matter collapses to form an uncharged frozar of mass $M$ and surface tending to the gravitational radius $r_{b} \rightarrow r_{g}$. At the same time, the charged particles on the surface do not move along geodesics, and each of them is subject to the Coulomb repulsion force generated by the entire charge of the ball, collected on a thin charged shell. Therefore, at collapse with the formation of a frozar, this charged shell lags behind the neutral matter of the ball and freezes at a slightly higher distance $r_{b} \rightarrow r_{g}+h$ with $h \ll r_{g}$. Thus, the charged frozar consists of an uncharged frozar, above the frozen surface of which a charged thin shell is frozen at very small distance [11]. However, here we will neglect such a fine structure of the charged frozar.

As the collapsed ball passes into the frozar state with $r_{b} \simeq r_{g}$, the ball's external metric (11) transforms to the frozar's external metric $\left(r>r_{g}, r>Q^{2} / r_{g}\right)$ :

$$
e^{v}=e^{-\lambda} \simeq 1-\frac{r_{g}}{r}-\frac{Q^{2}}{r_{g} r}\left(1-\frac{r_{g}}{r}\right)=\left(1-\frac{r_{g}}{r}\right)\left(1-\frac{Q^{2}}{r_{g} r}\right) .
$$

Outside the frozar the spacetime interval (5), therefore, takes the form:

$$
d s^{2} \simeq\left(1-\frac{r_{g}}{r}\right)\left(1-\frac{Q^{2}}{r_{g} r}\right) c^{2} d t^{2}-\left(1-\frac{r_{g}}{r}\right)^{-1}\left(1-\frac{Q^{2}}{r_{g} r}\right)^{-1} d r^{2}-r^{2} d \Omega^{2} .
$$

Introducing the notation $\Delta=r^{2}-\left(r_{g}+Q^{2} / r_{g}\right) r+Q^{2}$, we write the Eqs. (12) - (13) as:

$$
\begin{gathered}
e^{v}=e^{-\lambda} \simeq \frac{\Delta}{r^{2}}=1-\frac{r_{g}+Q^{2} / r_{g}}{r}+\frac{Q^{2}}{r^{2}} . \\
d s^{2} \simeq \frac{\Delta}{r^{2}} c^{2} d t^{2}-\frac{r^{2}}{\Delta} d r^{2}-r^{2} d \Omega^{2} .
\end{gathered}
$$

From (13) it can be seen that for external observers $r_{g}$ appears as the gravitational radius of not only the neutral matter, but also of the entire charged frozar, i.e. when the surface is asymptotically approached $r_{g}$, i.e. $r_{b} \rightarrow r_{g}$, all processes gravitationally freeze in terms $t$. This is due to the fact that, near the surface, the contribution to the metric comes from the energy of the electric field from a very thin layer $r_{b}-r_{g}=h \ll r_{g}$, which is insignificant. Therefore, the gravitational radius of a charged frozar practically is equal to the gravitational radius of its matter $r_{g}$, as a result of which the gravitational radius $r_{g}$ of the charged frozar appears in its metric (12).

Thus, the frozar's metric (12) includes only two parameters $r_{g}, Q$ and these parameters can change independently, i.e. the metric (12) is a metric with independent parameters. On the 
contrary, the RN metric (1) is a metric with dependent parameters, since at $r>r_{g}$ its parameters $\bar{r}_{g}$ and $Q$ are related by Eq. (4). As it will be shown in Section 4, the ignoring of this dependence in the black hole theory led to the set of physically absurd predictions.

Introducing the frozar's "charge radius" $r_{e}$ :

$$
r_{e}=\frac{Q^{2}}{r_{g}}=\frac{e^{2}}{2 M c^{2}}
$$

the frozar metric (12) can also be written in the form:

$$
e^{v}=e^{-\lambda} \simeq \frac{\Delta}{r^{2}}=\left(1-\frac{r_{g}}{r}\right)\left(1-\frac{r_{e}}{r}\right)=1-\frac{r_{g}+r_{e}}{r}+\frac{r_{g} r_{e}}{r^{2}},
$$

where $\Delta=r^{2}-\left(r_{g}+r_{e}\right) r+r_{g} r_{e}$. In this case, the interval (13) takes the form:

$$
d s^{2} \simeq\left(1-\frac{r_{g}}{r}\right)\left(1-\frac{r_{e}}{r}\right) c^{2} d t^{2}-\left(1-\frac{r_{g}}{r}\right)^{-1}\left(1-\frac{r_{e}}{r}\right)^{-1} d r^{2}-r^{2} d \Omega^{2}
$$

In the general case of a charged ball, the metric (11) can also be written in terms of $r_{e}$ :

$$
e^{v}=e^{-\lambda} \simeq 1-\frac{r_{g}}{r}-\frac{r_{g}}{r} \frac{r_{e}}{r_{b}}\left(1-\frac{r_{b}}{r}\right)=1-\frac{r_{g}}{r}\left[1+\frac{r_{e}}{r_{b}}\left(1-\frac{r_{b}}{r}\right)\right], \quad r \geq r_{b}
$$

For stars, the value of $r_{e}$ is negligible, while for elementary particles, it is negligible the value of $r_{g}$. These two radii are equal $r_{e}=r_{g}$ only when $M^{2}=e^{2} / 2 G$. For particles, as a rule, $r_{e}>r_{g}$ and if the effective "size" of a particle is considered to be of the order $r_{e}$, i.e. $r_{b}=r_{e}$, then (19) turns into:

$$
e^{v}=e^{-\lambda} \simeq 1-\frac{r_{g}}{r}-\frac{r_{g}}{r}\left(1-\frac{r_{e}}{r}\right)=1-\frac{r_{g}}{r}\left(2-\frac{r_{e}}{r}\right), \quad r \geq r_{e}>r_{g}
$$

For the Planck energy particles of with $r_{g}=\hbar / M_{p l} c$ and $r_{e}=e^{2} / M_{p l} c^{2}$, by assuming $r_{b}=r_{g}$, we have $r_{e} / r_{g}=e^{2} / \hbar c=\alpha$ with $\alpha \simeq 1 / 137$, and $r_{e}$ is sufficiently less than $r_{g}$. The metric (14), or (17), in the first approximation, when $r_{g} \ll r$ and $r_{e} \ll r$, has the form:

$$
e^{v}=e^{-\lambda} \approx 1-\frac{r_{g}+r_{e}}{r}=1-\frac{r_{g}+Q^{2} / r_{g}}{r} .
$$

This approximate metric is useful for describing the effects at large distances.

\section{The time dilation factor and redshifts near compact charged sources}

The equations of motion for a particle of mass $m$ in a static gravitational field with the metric (5) under the condition $e^{v(r)}=e^{-\lambda(r)}=\Delta / r^{2}$ and their solutions for geodesic trajectories, expressed in terms of $\Delta$, are well known (see $[4,5,10,14])$. The metric for frozar (14), or (17), is only a special case with a specific expression for $\Delta$. Thus, the results should be the same as for the RN metric (1), but with the value of the constant $\bar{r}_{g}=r_{g}+Q^{2} / r_{g}$ as 
in (4). Therefore, in this and the next sections, we present only the results for the dependence on $Q$ of the observable effects, referring to the literature for details (see $[4,5,10]$ ).

At a point $r>r_{g}$ near frozar, the decrease in the proper time interval $\Delta \tau$ between two events with respect to the world time interval $\Delta t$ between the same events is given by the expression $\Delta \tau=\Delta t g_{00}^{1 / 2}(r)$. The explicit form of the time dilation factor $g_{00}^{1 / 2}(r)$, according to (12), is given by the expression:

$$
g_{00}^{1 / 2}(r)=e^{v / 2}=\left(1-\frac{r_{g}}{r}\right)^{1 / 2}\left(1-\frac{Q^{2}}{r_{g} r}\right)^{1 / 2} \approx 1-\frac{1}{2 r}\left(r_{g}+\frac{Q^{2}}{r_{g}}\right)+O\left(r^{-2}\right) .
$$

As we can see, at a large distance the time dilation increases (i. e. the factor $g_{00}^{1 / 2}(r)$ decreases) as $Q^{2}$. The plots of the dependence near the frozar at radius $r=3 r_{g}$ is shown in Fig. 5. It can be seen from these plots that qualitatively the dependence for a frozar is the same as for a ball, but the time dilation is stronger for the frozar than that for any ball. The fact is that the radius of the frozar's surface $r_{b} \simeq r_{g}$ is the smallest for balls with a given mass, which means that the total energy of the electric field $Q^{2} / 2 r_{b}$ (for a given charge) is the largest.

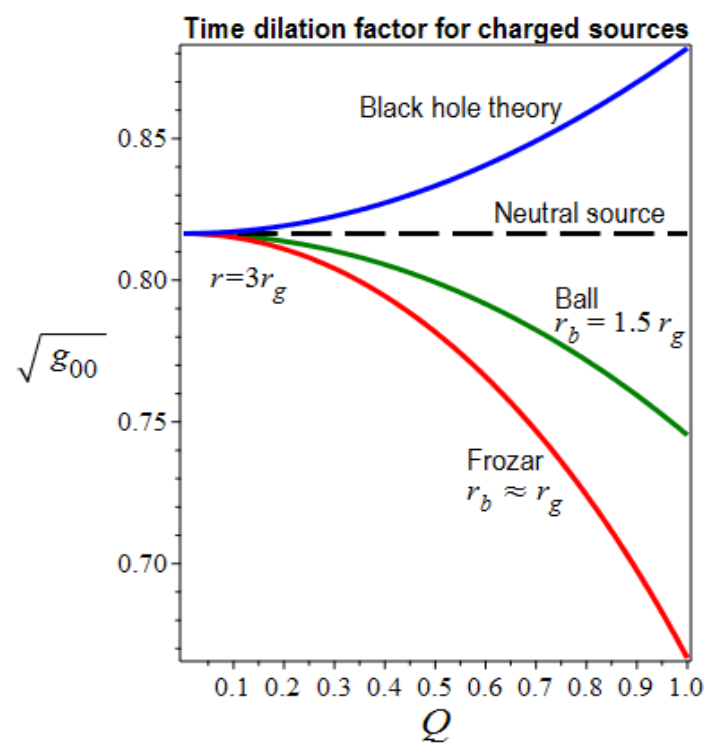

Fig. 5. Dependence of the time dilation factor $g_{00}^{1 / 2}(r)$ on the charge for a ball $\left(r_{b}=1.5 r_{g}\right)$, frozar $\left(r_{b} \simeq r_{g}\right)$ and a black hole at $r=3 r_{g}$.

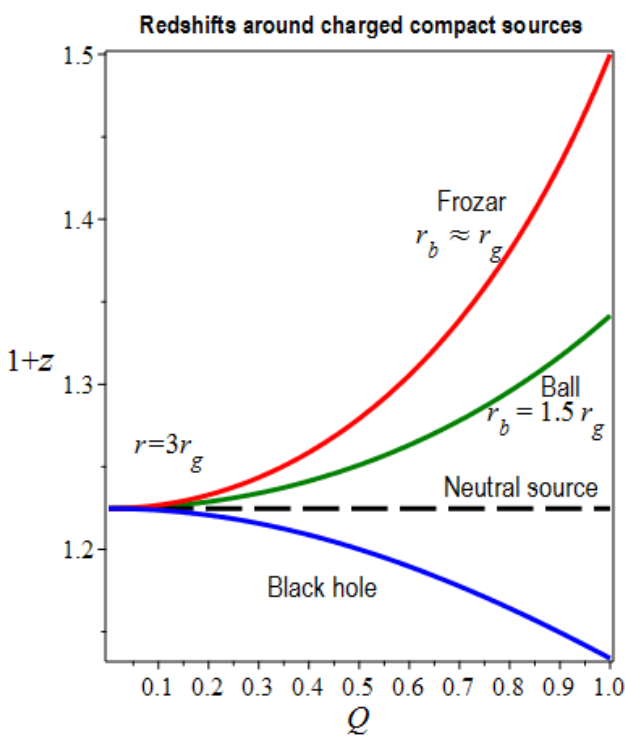

Fig. 6. Dependence of the redshift factor $1+z=g_{00}^{-1 / 2}(r)$ on the charge for a ball, a frozar, and a black hole at $r=3 r_{g}$.

Photons emitted at point $r$ near the frozar with natural frequency $\omega_{0}$ are received by a distant observer with a lower frequency $\omega<\omega_{0}$ and the redshift factor $1+z=g_{00}^{-1 / 2}(r)$, according to (12) and (22), is given by the expression:

$$
1+z=\frac{\omega_{0}}{\omega}=e^{-v / 2}=\left(1-\frac{r_{g}}{r}\right)^{-1 / 2}\left(1-\frac{Q^{2}}{r_{g} r}\right)^{-1 / 2} .
$$


Thus, the redshift of photons increases due to the contribution of the electric field energy to the gravitational field of the frozar along the entire path of the photon, and the redshift increases with increasing $Q$. Figs. 5-6 show this dependence in the two treatments.

\section{Radii of orbits and shadow}

The radii of the photon orbit $r_{p h}$, the innermost stable circular orbit $r_{I S C O}$ (for a particle of nonzero mass) and the radius of the shadow $\lambda_{c}$ are described by the same formulas as in the case of the RN metric (1) [5,10], but with the replacement $\bar{r}_{g}=r_{g}+Q^{2} / r_{g}$ as in (3)-(4). The radius of the photon orbit $r_{p h}$ is therefore equal to:

$$
r_{p h}=\frac{3}{4}\left(r_{g}+\frac{Q^{2}}{r_{g}}\right)\left(1+\sqrt{1-\frac{32 Q^{2}}{9\left(r_{g}+Q^{2} / r_{g}\right)^{2}}}\right) .
$$

The radius $r_{I S C O}$ is given by:

$$
\begin{aligned}
& r_{I S C O}=\frac{1}{2}\left(r_{g}+\frac{Q^{2}}{r_{g}}\right)\left[2+\xi+\frac{1}{\xi}\left(4-\frac{12 Q^{2}}{\left(r_{g}+Q^{2} / r_{g}\right)^{2}}\right)\right], \\
& \xi=4\left[2+\frac{8 Q^{4}}{\bar{r}_{g}^{4}}-\frac{Q^{2}}{\bar{r}_{g}^{2}}\left(9-\sqrt{\left(1-\frac{2 Q}{\bar{r}_{g}}\right)\left(5-\frac{8 Q}{\bar{r}_{g}}\right)}\right)\right]^{1 / 3},
\end{aligned}
$$

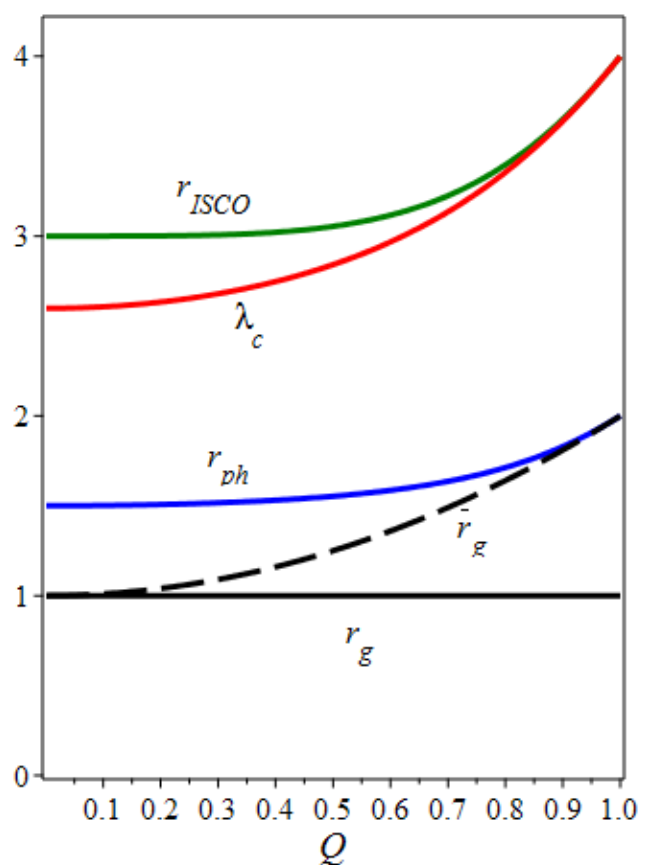

Fig. 7. The radii of the orbits and shadow around a charged frozar.

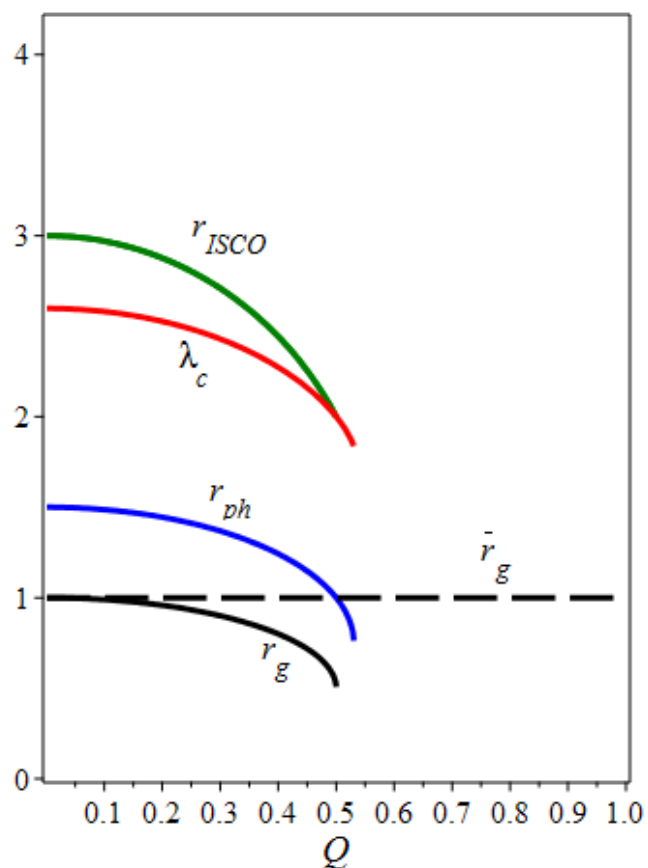

Fig. 8. The radii of the orbits and shadow around a charged black hole $[5,10]$. 
The radius of the shadow $\lambda_{c}$ of a frozar for a distant observer is:

$$
\lambda_{c}=\frac{r_{p h}}{\sqrt{g_{00}\left(r_{p h}\right)}}=\frac{3}{4}\left(r_{g}+\frac{Q^{2}}{r_{g}}\right) \cdot \frac{1+\sqrt{1-32 Q^{2} / 9\left(r_{g}+Q^{2} / r_{g}\right)^{2}}}{\sqrt{\left(1-r_{g} / r_{p h}\right)\left(1-Q^{2} / r_{g} r_{p h}\right)}} .
$$

Thus, at increasing $|Q|$, the gravitational radius of the frozar practically does not change: $r_{g}=$ const , and it follows from the expressions (24)-(27), that the radii $r_{p h}, r_{I S C O}$ and $\lambda_{c}$ grow almost as $Q^{2}$. The plots according the formulas (24)-(27) are presented in Figs. 7-8.

\section{Conclusion}

The external metric of the charged dust ball in the rest frame of the ball's center is static and follows from the Einstein equations on the hypersurfaces of simultaneity $t=c o n s t$. It was obtained earlier $[7,8]$ under the assumption about some distribution of the charge in the ball. In this case, the contribution of the electric field energy to the metric depended on an arbitrary function - the internal charge density. This charge distribution was introduced in the hope that the contribution of the electric field could stop the collapse.

As it is shown in the present article, these hopes were illusory for two reasons. First, in a real spherical star, charges will quickly collect on the surface, and the interior of the star, remaining electrically neutral, will continue to collapse. For this reason, in the article it is found a metric for a ball with a surface charge only, which is not only more realistic, but also much simpler, since the gravitational radius $r_{g}$ ceases to depend on the charge $Q$. Secondly, the contribution of the electric field energy to the metric, if taken into account correctly, has the same sign as the contribution of matter and therefore does not reduce, but enhances gravity in outer region. It is shown that as a result of this, the observable effects of gravity are also increases, in particular, at increasing $Q$, the radii of orbits and shadows, redshifts and angles of deflection of particles will increase.

Since, in Einstein's theory of gravity, the collapse of a dust ball leads to the formation of a frozar, a star with a gravitationally frozen structure and with a surface tending to the gravitational radius of matter $[11,12,14]$, the external metric for the frozar follows from the ball's metric when the surface tends to the gravitational radius $r_{b} \rightarrow r_{g}$. Thus, the frozar metric contains not three parameters $r_{b}, r_{g}$ and $Q$ as the ball, but only two ones $r_{g}, Q$. The observable consequences of the frozar metric are the same as for the ball, but with $r_{b} \simeq r_{g}$.

In the black hole theory with the RN metric for a point charge (1), the dependence on $Q$ of $\bar{r}_{g}$, (or total mass) appearing in the RN metric, was ignored. The result of this error was unphysical predictions that there are two event horizons, and the growth of the charge weakens the gravity of the black hole by reducing the observable effects of gravity, such as redshifts, radii of orbits and shadows $[5,10]$ etc. However, even after correcting this error, i.e. taking into account the dependence $\bar{r}_{g}(Q)$ according (4), the black hole theory will remain internally contradictory, since the absence of a surface, where the charge would be located, excludes the cutoff of the electric field at the lower limit. Since the electric field energy diverges for a point charge, the total mass of the entire black hole will also diverge. All this once again confirms the fact that the black hole theory is an abstract mathematical model, far from physical reality, and it is not applicable to astrophysical objects, the surfaces of which, at collapse, will remain outside their gravitational radii in any moment of cosmological time (see Appendix). 
The developed in the article method of reducing the metric to independent parameters can be applied to sources with angular momentum also and this is considered in the second article [12]. A more detailed study of the consequences of the new metric and further applications of the method to other metrics will be presented in the book [14].

\section{Appendix. The unphysical character of the black hole theory and its consequences}

As the charge grows, the gravitational radii of the ball and frozar practically do not change: $r_{g}=$ const, while the radii $r_{p h}, r_{I S C O}$ and $\lambda_{c}$, grow almost like $Q^{2}$, as can be seen in Figs. 5-8. The plots of the dependences predicted in black hole theory are also shown in the Figs. 58, and they show examples of non-physical consequences for observable effects. As $Q$ increases, which increases the electrostatic energy in the sphere of radius $r$, the time dilation factor $g_{00}^{1 / 2}(r)$ becomes closer to unity, and therefore the time dilation weakens, and redshifts decrease. The radii of characteristic orbits and the shadow of a black hole also decrease.

All these consequences mean the prediction of the anti-gravitational effect of the positive electrostatic energy and therefore are physically unacceptable. The fact that they are completely opposite to the predictions for an ordinary ball with a surface charge proves the erroneousness of these predictions of the black hole theory. In this regard, below we will consider in more detail the reasons due to which such unphysical consequences arose.

The effective gravitational radius $\bar{r}_{g}$ of a compact source with mass equal to the total mass $\bar{M}$ of a charged source, does not correspond to the value of the areal radius $r_{g}$ of a charged source at which the time component of the metric vanishes, i.e. is not its gravitational radius. Therefore, in the RN metric (1), the gravitational radius $r_{g}$ of the source, determined from the condition $e^{v\left(r_{g+}\right)}=0$, does not appear explicitly. In the black hole theory, it is stated that the time component of the RN metric (1) vanishes at two gravitational radii $r_{g( \pm)}[3,5,10]$ :

$$
r_{g( \pm)}=\frac{\bar{r}_{g}}{2} \pm \sqrt{\left(\frac{\bar{r}_{g}}{2}\right)^{2}-Q^{2}}
$$

If we follow (28), then the absolute value of the charge turns out to be limited as:

$$
|Q| \leq \frac{\bar{r}_{g}}{2}
$$

In contrary, as it shows the analysis of the metric of the ball with the surface charge, and the frozar as its limiting state at the collapse, it has a physical meaning in (28) only $r_{g(+)}$. According (28), at $Q \rightarrow 0$, non-zero value of $\bar{r}_{g}$ is equal to $r_{g(+)}$ and, therefore, the latter $i s$ the gravitational radius of uncharged matter $r_{g}$. Rewriting (28) in a convenient form and then expressing $\bar{r}_{g}$ in terms of $r_{g(+)}$, we obtain:

$$
\left(r_{g(+)}-\frac{\bar{r}_{g}}{2}\right)^{2}=\left(\frac{\bar{r}_{g}}{2}\right)^{2}-Q^{2}, \quad \bar{r}_{g}=r_{g(+)}+\frac{Q^{2}}{r_{g(+)}},
$$

which, according to (4) and (14), proves once confirms the identification $r_{g(+)}=r_{g}$. 
For a dust ball, as well as a frozar as its gravitationally frozen state, there is only one gravitational radius $r_{g}$ of matter with mass $M$, and the electric field energy, distributed in entire outer region, practically does not contribute to $r_{g}$. The constant $\bar{r}_{g}$ appearing in the RN metric is only the effective gravitational radius of an imaginary compact source with a mass equal to the total mass of the charged source at infinity $\bar{M}$, including the total energy of the electric field and is expressed through $r_{g}$ as in (4) or (30).

The statements of the black hole theory about a complicated structure of spacetime of a collapsed charged source, associated with the presence of two event horizons, a naked singularity, etc. $[3-6,10]$, thus turn out to be myths that arose due to the neglecting of the physical meaning of the total mass of this source.

The predictions made in the framework of the black hole theory on the basis of the $\mathrm{PH}$ metric (1) and shown in Fig. 5-8 are therefore the result of an erroneous assumption about constancy $\bar{r}_{g}$ at changing $Q$, while in reality $\bar{r}_{g}$, according (4), grows as $Q^{2}$, while $r_{g}$, the gravitational radius of neutral matter, remains constant.

Although this dependence was discovered a very long time ago, in 1960 [7, 8], and in 1971 an attempt was made to introduce it into the black hole theory in the form (4) [9], it has so far been ignored in this theory. This rejection was based on the fact that (4) implicitly assumes a cutoff of the electric field at the gravitational radius, while for a point charge, representing a charged black hole, there is no physical reason for such a cutoff. As a result, continuing to ignore the dependence $\bar{r}_{g}$ on $Q$, it was argued that the increase in $Q$, adding positive energy of the electric field, weakens the gravity of the black hole and, therefore, reduces the gravitational radius, redshifts, radii of the orbits and shadow [5,10], which is absurd.

The fact that this mistake has been systematically repeated in the gigantic literature on the black hole theory for more than half a century is not surprising in view of the abstractmathematical nature of this theory itself, which is far from physical reality. In fact, in Einstein's theory of gravity, as shown by the plots of the world lines of particles in the layers of a dust (uncharged) star [11], constructed according to the Oppenheimer-Snyder solution [12], the collapse leads to the formation not of a black hole, but of a frozar with a frozen internal structure, such as this structure was before freezing. The surface of the frozar in the rest frame of its center does not cross the gravitational radius in a finite cosmological time, and its layers asymptotically tend to their gravitational radii associated with the mass inside each layer.

In this regard, we note that, while recognizing purely formally the validity of such a treatment of the process of collapse of a neutral spherical star and its result in the form of a frozen star, followers of the black hole theory confidently declare that this picture is "apparent" and is related only with a "bad" choice of the time coordinate in the form of world time $t$. Further, it is argued that at introducing a more "suitable" time coordinate, the proper time $\tau$, the surface of the collapsing star "easily" crosses the gravitational radius of the star at a finite moment $\tau_{g}$, and that it is insufficient that this corresponds to the moment $t=\infty$ of the cosmological time in the vicinity of this star, which never happens in the real world. Then, the scenario of the Newtonian theory is described, according which at $\tau>\tau_{g}$, i.e. at $t>\infty$ (!), all the matter of the star quickly reaches the center $r=0$, forming a central singularity surrounded by an event horizon at $r=r_{g}$. Having thus outlined the formation of a black hole at $t>\infty$, it is immediately stated that the known collapsed stars with $M>3 M_{\circ}$ at a given moment of world time $t<\infty$ are actually existing black holes.

In the astrophysical literature, a careful clarification was sometimes made that the horizon of real collapsars "has not yet formed" and it was proposed to call them as "astrophysical black holes", distinguishing them from "real" ones with a horizon. This is a hidden recognition that 
the "horizon" of the real collapsars will never form, and, therefore, at any given moment of our time they represent a star with a frozen structure, i.e. frozar.

The above mentioned split of reality was not considered as a contradiction. Moreover, it was discussed in the literature such a question: where to get those half an hour after infinity, when a black hole is formed? The answer was an even more irrational statement that in this theory, besides our reality, there must be other realities and that in general we are dealing with surrealism here [15].

In fact, behind such far-reaching statements, which at first glance have a deep meaning, lie the same errors that arose due to carelessness in the physical meaning of the concepts and quantities used, which was revealed in the case of the charged source. The main one was the erroneous statement $[16,17]$, from which the wide recognition of this theory began, that in the well-known article of 1939 [12] Oppenheimer and Snyder proved that the surface of a spherical dust star collapses into the gravitational radius and quickly reaches the center $r=0$. As it was shown in [11] (see also [14]), firstly, there is no such statement in [12], and secondly, just the opposite was proved there, that the surface of the star freezes over $r_{g}$ and the asymptotics of the metric for the inner layers was found, as well as the solution for particle trajectories in these layers, showing that each layer also freezes above its own gravitational radius associated with the mass inside it.

A more detailed criticism of the theory of black holes and the proof of its inconsistency as a physical theory is given in [14].

\section{References}

1. Reissner H. (1916) Ann. d. Phys., 50, 106.

2. Weyl H. (1917) Ann. Phys., 359(18), 117.

3. Nordström G. (1918) Proc. Kon. Ned. Akad. Wet., 20, 1238.

4. Jeffery G. (1921) Proc. R. Soc. Lond. A99 (697) 123.

5. Bambi C. (2017) Black Holes: A Lab. for Testing Strong Gravity. Springer.

6. Einstein A., Rosen N. (1935) Phys. Rev., 48(1), 73.

7. Bonnor W. (1960) Z. Phys., 160, 59;

8. Florides P. (1960) PhD Thesis, U. London; (1962) Math. Proc. Cambr. Phil. Soc. 58(1) 110; (1977) Nuov. Cim., A42(3), 343.

9. Christodoulou D., Ruffini R. (1971) Phys. Rev., D4, 3552.

10. Pugliese D., Quevedo H., Ruffini R. (2011) Phys. Rev. D83(2), 024021.

11. Zakir Z. (2018) Astr. \& Space Sci. 363: 30; (2020) Quant. \& Grav. Phys., 1:006-7132; 1:007-7133

12. Oppenheimer R., Snyder H. (1939) Phys. Rev. 56(1), 455.

13. Zakir Z. (2022) Quant. and Grav. Phys., 3:017-7677.

14. Zakir Z. (2022) Theory of Frozars. Relativistic Collapse of Stars, CTPA, T.

15. Ne'Eman Y. (1994). Found. Phys. Lett. 7(5), 483.

16. Wheeler J. (1964) in Relativity, Groups and Topology, G. \& B. Publ.

17. Penrose R. (1965) Phys. Rev. Lett. 14(3), 57. 